\title{
Capital social y gestión participativa en la cuenca de Pátzcuaro
}

\author{
John Durston y Eduardo López
}

$\mathrm{P}$

artiendo de un recuento teórico de los postulados del capital social, se analiza aquí la utilidad y alcances que este enfoque tiene para generar soluciones que puedan revertir los procesos de deterioro ambiental, basándose en la activación y potenciación del capital social existente en diversas comunidades y grupos sociales. Para tal efecto, se toma como referente empírico la situación socioambiental de la cuenca del Lago de Pátzcuaro y se explora los atisbos sociales y políticos de reemergencia del capital social en la zona, los procesos que permitirían desatar cambios significativos mediante el capital social, y los alcances y limitaciones a lo que una agencia externa puede hacer al respecto.

John Durston

Antropólogo

Consultor y Evaluador

Santiago de Chile

- jdurston@mi.cl

Eduardo López

Sociólogo, Investigador

del Instituto Mexicano de Tecnología

del Agua (IMTA),

Subcoordinación de Participación Social

$\propto$ elopez@tlaloc.imta.mx 


\section{I}

\section{Introducción}

En América Latina, la relación entre el Estado y las comunidades locales se ve gravemente afectada por la debilidad actual de la sociedad civil y por problemas como la corrupción (Peña y Solanes, 2003). Según Hooper (2001), a pesar de estas dificultades y de la falta de confianza de la gente en sus gobiernos, es posible crear comunidades comprometidas con el futuro de su región mediante una combinación de líderes motivados y un trabajo 'cara a cara' en redes.

En este contexto, el debate teórico sobre el capital social — que abarca una gran cantidad de temas relacionados con la interacción del Estado y la sociedad civil- se ha convertido en un nuevo referente para analizar problemas como la superación de la pobreza, la participación social y el desarrollo rural, con miras a aportar soluciones prácticas mediante la potenciación del capital social en diversos grupos o comunidades. Además, nosotros consideramos que este enfoque puede significar un acercamiento interesante al análisis de la gestión integral de las cuencas hidrográficas.

En virtud de lo anterior, este trabajo toma como referente empírico la situación socioambiental de la cuenca del Lago de Pátzcuaro para plantearse las siguientes interrogantes: ¿sobre qué nuevas bases se puede iniciar una verdadera recuperación ambiental de la zona que garantice una mejor calidad de vida y un crecimiento económico, sin atentar contra la permanencia y reproducción de los recursos naturales?, ¿qué puede hacer una agencia externa para fortalecer la participación en sectores subordinados?

Para responder a estas preguntas, primero se pasa revista a algunos aspectos pertinentes de la teoría del capital social; segundo, se analiza la situación de la cuenca, el quehacer de los actores sociales y las posibilidades de construir, reconstruir o potenciar el capital social en dicha zona, y tercero, se presentan algunos atisbos de mejoramiento de la situación.

\section{II}

\section{El debate sobre el capital social}

El capital social no es una receta, ni siquiera un marco teórico consensuado, sino un debate ${ }^{1}$ en marcha, transdisciplinario y holístico, en el que participan con un mismo lenguaje economistas, sociólogos, antropólogos y politólogos. La evolución de este debate obliga a reevaluar algunas ideas establecidas sobre las conductas sociales.

\section{1. ¿Qué es el capital social y cómo funciona?}

Hay una visión relativamente consensuada de que las personas y los grupos son actores, agentes o sujetos que tratan de llevar a cabo proyectos de vida y emprendimientos con diversos objetivos, y que para realizar sus estrategias movilizan activos, incluidos activos

\footnotetext{
${ }^{1} \mathrm{Al}$ respecto hay gran cantidad de documentos recientes en español en el sitio web del Banco Mundial (www.bancomundial.org) y, con una visión un poco diferente, en el sitio de la CEPAL (www.cepal.cl).
}

intangibles como el capital social (Bebbington, 2005). Adler-Lomnitz (2003 y 1998, respectivamente) ha mostrado la importancia de la movilización de activos que hacen los grupos marginados para sobrevivir, y la clase media chilena para obtener favores; en ambos casos la formación o existencia de redes de intercambio resulta fundamental; sin duda, los mecanismos identificados por ella guardan una estrecha relación con las diferentes formas de capital social, así como con las estrategias de movilidad de los actores sociales.

Entre las múltiples visiones diferentes sobre el capital social, adoptamos aquí la visión selectiva de la CEPAL: el capital social es el contenido de relaciones sociales y de instituciones sociales, basado en la reciprocidad difusa y caracterizada por prácticas repetidas de cooperación que generan confianza (Atria, Siles y otros, 2003). En esta definición, el capital social está analíticamente separado del capital cultural, pero se tiene presente que ambos cambian permanentemente, en constante retroalimentación. 
El capital social ha estado presente en la sociología durante décadas: Bourdieu y Coleman utilizaron esta denominación ya en la década de 1980, mientras que Granovetter (1985) habló de "lazos fuertes y lazos débiles", y North (1990) desarrolló su teoría de institucionalidad con contenidos muy similares a lo que hoy se llama capital social. El concepto se puso de moda en el debate sobre el desarrollo, y especialmente sobre el papel de la sociedad civil en el desarrollo, a partir de la publicación de un libro (Putnam, 1993) que celebró el papel del capital social en la regionalización de la política pública en ciertas áreas de Italia.

En un artículo en World Development, Jonathan Fox se pregunta cómo se densifica la sociedad civil regional (Fox, 1996). En su análisis echa mano al concepto de capital social e inventa otro: el semiclientelismo. Lo que le interesa es saber cómo aumentan de escala en México las pequeñas organizaciones locales para formar una red activa de asociaciones civiles y movimientos sociales a nivel regional. Pero nuestra preocupación en este trabajo ha tenido que partir de un nivel más elemental: qué hacer en regiones como Pátzcuaro donde, pese a su fuerte tradición comunitarista ${ }^{2}$ y organizativa, los intentos de "desarrollo" no han prosperado o se han dispersado en programas que constituyen prebendas del clientelismo autoritario, convirtiendo a muchas organizaciones en receptoras pasivas de prácticas clientelistas. Lo que parece lógico en situaciones como esta (tan comunes en América Latina) es bajar la escala de análisis y acción, en lugar de elevarla, para mirar las unidades más pequeñas de capital social, es decir, los vínculos entre individuos. Este es un primer paso necesario incluso en una región tan grande y tan politizada como la cuenca del Pátzcuaro, porque son los vínculos interpersonales, fortalecidos por principios de reciprocidad, los que mueven la institucionalidad informal y, por ende, el quehacer cívico a mayor escala.

\section{Elementos, dinámicas y tipos de capital social}

Adler-Lomnitz (2003) y Mauss (1979), entre otros antropólogos, han demostrado que la reciprocidad, el intercambio y la confianza son la base de toda interacción humana sostenida destinada a iniciar o a fortalecer relaciones sociales. Dichas actitudes implican una obligación de retribuir en el futuro a la otra parte (el socio) y estar a su

\footnotetext{
${ }^{2}$ Con este término nos referimos al fuerte apego que los pobladores tienen a su comunidad y a su sentido de pertenencia a la misma.
}

disposición, sin que esto signifique llevar una cuenta. Su base es el 'contrato diádico', concepto desarrollado por el antropólogo George Foster tras varios años de estudio de la zona de Pátzcuaro (Foster, 1963). La multiplicación de los contratos diádicos genera redes egocentradas, cuya intensidad de intercambio se basa en cuatro factores: i) la distancia social; ii) la distancia física; iii) la distancia económica, y iv) la distancia psicológica (Adler-Lomnitz, 2003). Participar en estas redes, a su vez, produce 'bienes socioemocionales' (Robison, Siles y Schmid, 2003) y refuerza los vínculos del capital social, que se acumula con el uso. La activación repetida de estos vínculos resulta en un aprendizaje colectivo sobre las posibilidades de cooperación y en un aumento de confianza, lo que eleva la capacidad de emprendimiento colectivo.

Partiendo de los contratos diádicos, los actores sociales reclutan aliados a partir de una matriz de parentesco y vecindad. De esta manera los grupos se multiplican para formar asociaciones de trabajo, comunidades y sociedades regionales con capital social. En esta transformación, los propietarios del capital social pasan de ser dos individuos a ser actores colectivos, $o$ a ser la sociedad en su conjunto.

En los últimos años ha habido un proceso de reconstrucción del concepto de capital social (recuadro 1). Este no solo da acceso a bienes escasos, sino que, según se reconoce actualmente, constituye en sí mismo un bien escaso. El capital social es un arma en un entorno socioeconómico en el que están también presentes la competencia, la rivalidad, los conflictos, la traición y el engaño.

Actualmente se reconoce que existen diferentes tipos de capital social. Los más conocidos son los de unión, de puente y de eslabón (bonding, bridging y linking). Este triple esquema, desarrollado para el Banco Mundial por Woolcock (1998), ha sido criticado por concebir 'niveles' de capital social sin reconocer la existencia de desigualdades de poder. Como ha señalado Fine (2001), en el Banco Mundial olvidaron a Bourdieu (2001), para quien el uso de capital social de las elites nacionales es principalmente un instrumento de subordinación, extracción y exclusión. No se debe caer en la visión romántica de que el capital social siempre es bueno para la sociedad toda. Pero es bueno tenerlo.

Se han generado diversas tipologías sobre el capital social (Durston, 2002; Arriagada, Miranda y Pávez, 2004), que pueden contribuir a su estudio empírico en situaciones concretas. Sin embargo, dichas tipologías no pueden encasillarse en la realidad, y debemos utilizarlas solo como instrumentos de análisis para identificar las distintas formas de capital social y analizar las posibilidades de potenciarlo. 


\author{
Recuadro 1 \\ DEFINICIONES DE CAPITAL SOCIAL ${ }^{\mathrm{a}}$
}

\begin{abstract}
Robert Putnam
Por capital social entendemos aquí la confianza, las normas que regulan la convivencia, las redes de asociacionismo cívico, elementos que mejoran la eficiencia de la organización social promoviendo iniciativas tomadas de común acuerdo (traducido de Putnam, 1993).

James Coleman $\quad$ No es una entidad aislada, sino una variedad de entidades que tienen dos características en común: consisten en algún aspecto de la estructura social, y facilitan a los individuos que están dentro realizar ciertas acciones (traducido de Coleman, 1990).

Pierre Bourdieu

Es el agregado de los actuales o potenciales recursos que están relacionados con la posesión de una red perdurable de relaciones más o menos institucionalizadas de conocimiento y reconocimiento mutuo -en otras palabras, con la pertenencia a un grupo- que le brinda a cada uno de los miembros el respaldo del capital socialmente adquirido, una credencial que les permite acreditarse, en los diferentes sentidos de la palabra (traducido de Bourdieu, 1980).

Francis Fukuyama "Puede ser definido, simplemente, como un conjunto de valores o normas informales compartidas entre los miembros de un grupo, que permiten la cooperación entre los mismos" (Fukuyama, 1999).

Banco Mundial El capital social se refiere a las normas y redes que disponen de acción colectiva. En incremento de las evidencia muestra que la cohesión social -capital social- es crítica para el alivio de la pobreza y el desarrollo sostenible, humano y económico. (Disponible en www.bancomundial.org).

John Durston

"Se entenderá por capital social el contenido de ciertas relaciones y estructuras sociales... las actitudes de confianza que se dan en combinación con conductas de reciprocidad y cooperación" (Durston, 2002).
\end{abstract}

Fuente: elaboración propia sobre la base de la bibliografía citada.

${ }^{\text {a }}$ Las definiciones de Putnam, Coleman y Bourdieu fueron proporcionadas en español por los autores de este artículo.

Uno de los avances en la tipología del capital social ha sido la distinción entre sus formas horizontal $\mathrm{y}$ vertical. En sentido estricto, no existen las relaciones puramente horizontales, porque incluso en comunidades y familias pobres hay importantes diferencias en el grado de control que se tiene sobre las relaciones sociales de sus integrantes. Todas las relaciones sociales son en alguna medida verticales y asimétricas. La expresión "capital social horizontal" es una forma abreviada de referirse a vínculos que existen dentro de un mismo estrato social, y la expresión "capital social vertical" se refiere a vínculos entre estratos, clases o castas, o entre "patricios' y 'plebeyos".

Otra vez fue Foster (1963) quien señaló que una forma importante del contrato diádico es la relación patrón-cliente, ejemplificado en el santo patrón de la comunidad, al cual se rinde tributo y que concede favores. Justamente los cargos sociorreligiosos de los cofrades que auspician las fiestas de los santos patrones de barrios y comunidades en la región de Pátzcuaro ilustran cómo el enfoque de capital social obliga a cuestionar teorías establecidas (en este caso, de Foster y de otros). Todavía predomina entre los analistas la idea de que los gastos en fiestas sirven como mecanismos de nivelación de la riqueza y la posición social, al exigir mayores gastos y dedicación de tiempo a los hombres que han iniciado un proceso de acumulación.

Desde el enfoque del capital social, parece más bien que el gasto y la dedicación de tiempo a estas actividades aparentemente no productivas operan como inversiones en prestigio y en reciprocidad difusa, que posteriormente rinden no sólo un tejido social de reciprocidades reforzadas por su activación, así como prestigio para el cofrade, sino también beneficios económicos individuales. Los gastos asociados con los cargos no rebajan a quien los ostenta al nivel común de pobreza, como postulan estos críticos. Si bien durante el año que sigue al auspicio de un gasto cívico-religioso se reduce el capital (económico) de estas personas, a la larga se acrecienta su prestigio y su fortuna material gracias al aumento de la confianza, la capacidad de movilizar mano de obra y las posibilidades de pedir ayuda a socios.

Las redes de parentesco, activadas para organizar la fiesta, constituyen la principal base para la acumulación de capital material y social. Actualmente esas redes 
son activadas también para facilitar la migración, y los ahorros realizados a través de ella son reinvertidos por algunas comunidades en grandes fiestas religiosas. ${ }^{3}$ Los objetivos de los emigrantes retornados al realizar estos fuertes gastos incluyen (no debemos olvidarlo) el cumplimiento de promesas profundamente religiosas, sentidas genuinamente por los cofrades como una deuda con el santo.

A pesar de los embates de la economía capitalista, del clientelismo y de la emigración, estas prácticas son elementos de capital social colectivo aún presentes en forma "subsumida" (Salazar, 2001), que pueden ser rescatados de la memoria social y activarse en nuevos emprendimientos grupales, comunitarios e intercomunitarios. Hay, pues, un permanente juego entre el capital social individual y el colectivo. Además de pares de socios, las personas tienden a formar grupos con líderes, que son la base de la empresa y de la comunidad. Las mismas estructuras y formas de cooperación de estas instituciones sociales son recursos para lograr emprendimientos mayores, es decir, constituyen capitales sociales colectivos.

Pese al avance del debate sobre el capital social, Bebbington (2005) hace hincapié en los "silencios" y las áreas nuevas de reflexión sobre capital social: mujer, ingreso y poder. $\mathrm{Al}$ respecto, podemos comentar lo siguiente:

i) Capital social y mujer. En América Latina las mujeres ya no carecen de voz, sino que, por el contrario, constituyen uno de los pocos movimientos sociales hoy vigentes. En la zona de Pátzcuaro han sabido desempeñarse en los espacios que ha dejado la emigración de los hombres. Sin embargo, como advierte Mercedes González de la Rocha (2003), las líderes serviciales son mano de obra gratuita para muchos programas estatales que aprovechan su capital social en condiciones de extrema pobreza.

ii) Capital social e ingreso. Putnam (1993) estableció la moda de concentrarse en la "civilidad" del capital social. Sin embargo, estudios recientes, como el de Berdegué (2001), analizan las maneras en que el capital social campesino facilita el "cooperar para competir" en empresas asociativas, produciendo mejoras en los ingresos.

iii) Capital social y poder. El poder está ausente en la visión conservadora del capital social, y en el Banco

\footnotetext{
${ }^{3}$ Por ejemplo, existe información documentada sobre las redes de migrantes en Oaxaca y Michoacán. Ejemplos importantes de ellas son el Frente Indígena Oaxaqueño Binacional (FIOB) y la Federación de Clubes Michoacanos en Illinois.
}

Mundial donde Bebbington trabajó. También suele faltar en los discursos y las prácticas de programas para reducir la pobreza, donde la idea del conflicto de clases está ausente por razones políticas.

\section{Las estrategias de movilidad del capital social}

Quienes se han apoyado en los postulados teóricos del capital social, y han utilizado este concepto como un instrumento para impulsar los programas de desarrollo comunitario o de combate contra la pobreza, sostienen que él presenta dos dimensiones mediante las cuales los grupos o las comunidades pueden alcanzar sus objetivos comunes: i) la capacidad específica de movilización de determinados recursos por parte de un grupo, y ii) la disponibilidad de redes de relaciones sociales.

En ambas dimensiones la capacidad de movilización se hace presente a través de dos nociones distintas: el liderazgo y el empoderamiento, por lo que también se han identificado dos estrategias - no excluyentes - para desarrollar el capital social de un grupo:

i) La estrategia de asociatividad, por la cual las acciones grupales se orientan a fortalecer la trama de redes en las que participan los miembros del grupo a fin de potenciar sus lazos mediante nuevas relaciones. Aquí, los vínculos con otros grupos se conciben como una estrategia de cooperación y alianzas que, según Atria (2003), "está basada en el desarrollo del capital social, pasando de una situación de predominio de redes internas a otra situación de predominio de redes externas al grupo".

ii) Estrategia de movilización, que se basa en el desarrollo del capital social, pasando de una situación donde predomina el liderazgo en el grupo a otra situación donde predomina el liderazgo para el grupo. Atria (2003) considera que esta es una estrategia de empoderamiento, debido a que la influencia que algunos miembros del grupo tienen dentro de él se transforma en una organización que le permite al grupo actuar en su entorno con respecto a otros grupos.

Estas estrategias no están desvinculadas del aparato burocrático encargado de llevar a cabo los programas; por el contrario, existen redes burocráticas que facilitan o dificultan la obtención de resultados, ya que "los programas están integrados (embedded) en relaciones sociales. En el lenguaje del capital social, eso significa que las agencias de desarrollo y los agentes públicos proyectan redes burocráticas hacia el nivel comunitario. Tales relaciones sociales influyen a su vez en sus 
capacidades y forma de actuar" (Arriagada, Miranda y Pávez, 2004).

La manera en que cada programa entiende los problemas que intenta resolver, y la visión de quien lo diseña y lo pone en práctica, influyen también en la orientación del mismo, ya que: "Son las actitudes y las prácticas cotidianas de estos promotores, los factores que definen si un programa público logra crear lazos de confianza entre gobierno y ciudadano; y las redes sociales de estos promotores son factores determinantes de cómo los beneficios de estos programas se distribuyen a nivel local. En consecuencia, hay que entender de una manera matizada cómo el capital social de escalera (o sea, aquel que vincula al ciudadano con agentes externos) se forma y se mantiene y, por lo tanto, quiénes en la práctica tienen o no esta forma de capital social" (Arriagada, Miranda y Pávez, 2004).

Con todo lo dicho aquí, podemos afirmar que el enfoque del capital social conlleva varias ideas que tienen importancia y utilidad para la forma en que tradicionalmente se ha abordado la gestión integral del agua:

- Permite entender la organización más allá de los cánones formales, es decir, como simples agregados de individuos que tienen como función avalar los programas gubernamentales.

- No es necesario establecer un organismo especial para participar en actividades orientadas a la recuperación de un espacio geográfico - como una cuenca o una región-, pues es la convergencia de intereses y el beneficio común lo que guiará la participación y organización.

- La "inversión" en capital social, por lo tanto, puede generar mucho más resultados que la participación social tradicional y por ello los "cambios en la estructura de las relaciones sociales pueden tener efectos significativos en la distribución del poder y en una gama de otros recursos" (Bebbington, 2005).

- La "estructura de estas relaciones sociales influye en el funcionamiento tanto de las instituciones públicas como de las instituciones económicas (mercados, etc.) y, por lo tanto, los cambios en las relaciones sociales pueden llevar a cambios en el funcionamiento de tales instituciones" (Bebbington, 2005).

\section{4. ¿Qué pueden hacer las agencias externas para aumentar el capital social popular con miras a la planificación participativa?}

Con los elementos antes señalados, cabría preguntarse si el capital social es condición suficiente para el desarro- llo local o comunitario. La respuesta es negativa, pues no se debe olvidar que las relaciones son importantes para alcanzar el desarrollo local, pero que también lo son el capital físico y financiero (Trigilia, 2003). Cabe preguntarse entonces qué pueden hacer las agencias externas para aumentar el capital social, orientándolo a una participación que contribuya a revertir el deterioro ambiental, como en el caso que nos ocupa.

Una autoridad de cuenca con reglamentación, poder, recursos y beneficios sería ideal para estimular la acumulación de capital social. Pero la falta de ella en Pátzcuaro obliga a empezar la reconstitución del capital social dañado antes de esperar a que se establezca tal autoridad formal. Este puede ser incluso un paso previo para crear una demanda social por la creación legal de una entidad de gestión de la cuenca.

Evitar la politización es ilusorio. El enfoque de la CEPAL $^{4}$ propone la estrategia de provocar transiciones rápidas en el sistema sociopolítico territorial, visto como un sistema adaptativo complejo que emerge de la evolución conjunta de las estrategias de todos los actores, de manera parecida a un ecosistema. Para lograr esto, es necesario que una agencia externa lleve a cabo una serie de acciones estratégicas:

- Bajar de escala para subir de escala: la permanencia de promotores que puedan entregar beneficios inmediatos a esfuerzos cooperativos reiterados para regenerar la confianza dañada a nivel microlocal es el punto de partida para apoyar la reaparición de actores sociales populares autónomos en el sistema municipal y estatal.

- Respaldar a actores populares embrionarios que pueden ser cooptados o eliminados por actores clientelistas autoritarios.

- Intervenir el sistema territorial municipal. La organización de comunidades y la agencia promotora de capital social colectivo son dos nuevos actores en el sistema, cuya irrupción produce una coevolución de estrategias y genera un shock en el sistema. Ellos consiguen aliados que idealmente incluyen al presidente municipal y a facciones reformistas dentro de partidos establecidos.

Contrariamente a la visión de Putnam (1993) de un modelo de equilibrio dual, las sociedades no tienden a estabilizarse en extremos de baja o alta presencia de capital social, sino que pueden cambiar de rumbo con rapidez, producto de estas dinámicas complejas.

\footnotetext{
${ }^{4}$ Véase numerosos documentos sobre el tema en el sitio web de la CEPAL (www.cepal.cl).
} 


\section{III}

\section{La cuenca de Pátzcuaro y el capital social: problemas ambientales}

La cuenca del lago de Pátzcuaro ha sido sometida largamente a estudios, análisis, proyectos, obras y programas de diversa índole, por lo que sería ocioso tratar de hacer un nuevo diagnóstico que aporte novedades a lo escrito hasta hoy. Por lo tanto, en esta sección solo nos referiremos brevemente a los principales recursos de la cuenca y los problemas ambientales que la aquejan. Más adelante, partiendo de las acotaciones teóricas mencionadas, trataremos de dilucidar qué puede hacer una agencia externa como el Instituto Mexicano de Tecnología del Agua (IMTA) para potenciar el capital social en esta zona.

La cuenca estudiada abarca parte de los municipios que circundan el lago del mismo nombre y que son los de Pátzcuaro, Quiroga, Erongarícuaro y Tzintzuntzan. Se estima que en ella se ubican 86 comunidades -77 rurales y nueve urbanas- que en su conjunto agrupan a una población de 118.733 habitantes (INEGI, 2000).

Por sus características fisiográficas, la cuenca combina diversos ambientes en los cuales se realizan diferentes actividades productivas, particularmente pesca, silvicultura y actividades agropecuarias. Durante los últimos veinticinco años, la deforestación, la urbanización, la expansión de la frontera agrícola, la pérdida de fertilidad de las tierras de cultivo, la erosión, el azolve del lago, la maleza acuática, la disminución de la pesquería y la contaminación del lago por descargas residuales, han originado la alteración e inestabilidad de la cuenca, y por ende, una baja de la calidad de vida de sus habitantes y un incremento del deterioro ambiental.

Entre los principales problemas ambientales de la cuenca cabe identificar los siguientes: ${ }^{5}$

Mapa 1

México: Cuenca del lago de Pátzcuaro

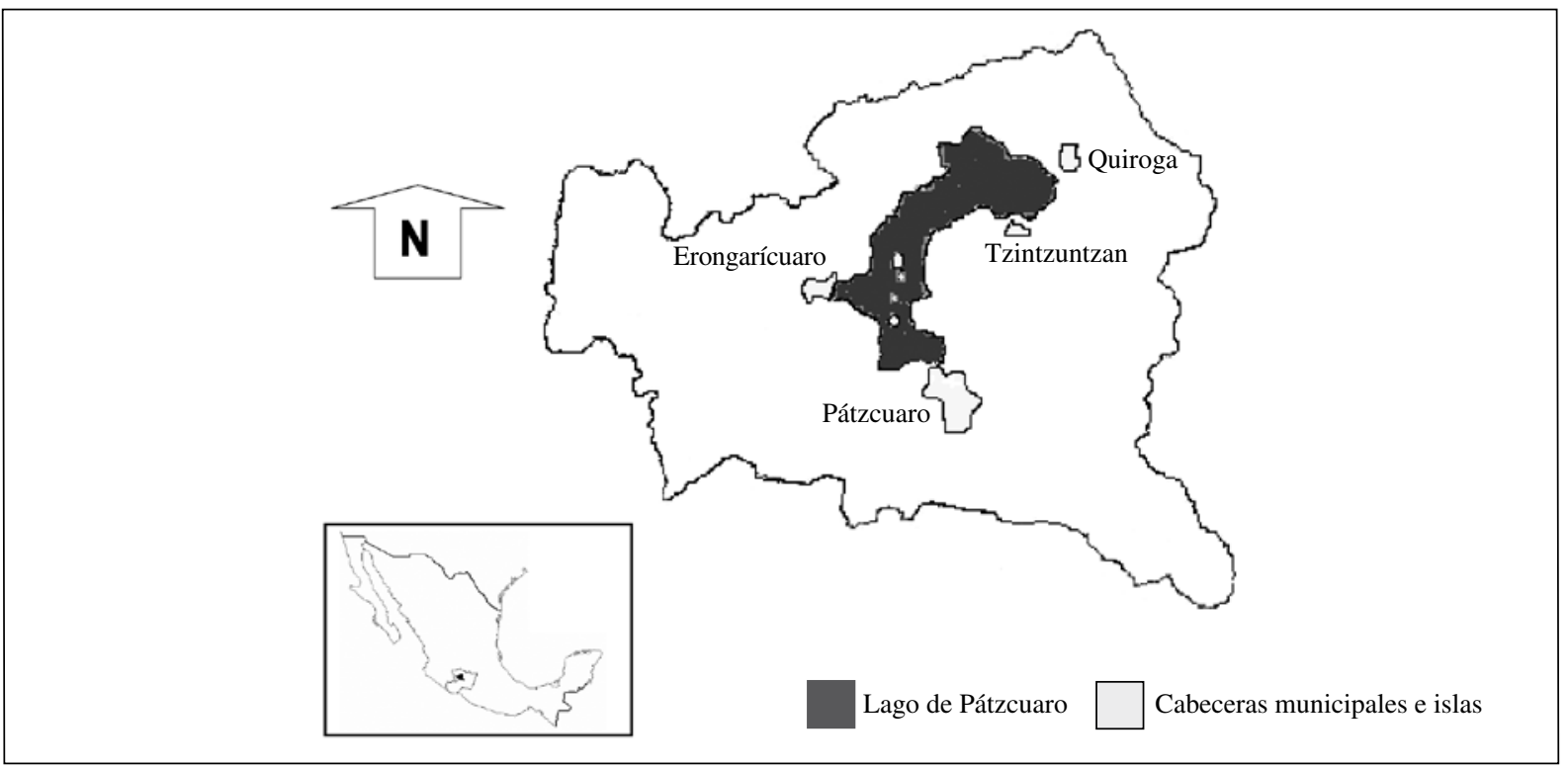

Fuente: elaboración propia.

${ }^{5}$ Salvo cuando se señala otra cosa, la información sobre los problemas de la cuenca del lago de Pátzcuaro, resumida en los cinco puntos que se enuncian, proviene del Atlas Cibernético de Pátzcuaro, que se puede consultar en http://www.centrogeo.org.mx/CiberAtlas/patzcuaro/. 
i) La erosión de los suelos. Es causada por diferentes factores, como la práctica agrícola de roza-tumbaquema, la ganadería extensiva en las áreas boscosas y los cambios de uso del suelo que, combinados con la existencia de suelos con textura muy ligera, pendientes pronunciadas, intensa precipitación pluvial y constantes incendios forestales, generan una acelerada pérdida de suelo.

ii) La deforestación. El $32 \%$ de la superficie de la cuenca corresponde a superficie arbolada que, si bien no es totalmente maderable, permite su múltiple utilización para extracción de resina, elaboración de artesanías, obtención de leña para uso doméstico, cocción de la alfarería, producción de madera en pie y su transformación en madera para fines comerciales en los aserraderos de la zona. Se estima que entre 1963 y 1991 la superficie arbolada ha disminuido a casi la mitad, debido a la tala clandestina, y que tres de cada cuatro metros cúbicos de madera que ingresan a los aserraderos de la región son clandestinos.

iii) El uso excesivo de fertilizantes en áreas agrícola. Aproximadamente el 25\% de la superficie de la cuenca tiene uso agrícola. Actualmente predomina la utilización de fertilizantes, sin que por ello haya aumentado significativamente la productividad de las tierras. Se estima que en la cuenca se aplican anualmente entre 5.000 y 10.000 toneladas de fer- tilizantes químicos. El acarreo de estos fertilizantes al lago, además de dañar el suelo, contribuye al proceso de eutroficación por sobrefertilización.

iv) Incremento desmedido de la actividad pesquera. En el lago se efectúa pesca artesanal, caracterizada por una escala de operación limitada, empleo de artes de pesca rudimentarias, elevado número de pescadores y poca capitalización. La pesca es realizada por 24 comunidades ribereñas e isleñas asentadas en los municipios que circundan el lago, lo que ocasiona una sobreexplotación de las especies de agua dulce. La Secretaría de Medio Ambiente y Recursos Naturales (SEMARNAT) estima que existen 2.000 pescadores, los que en 1989 capturaron 2.300 toneladas de pescado; la producción cayó a 634 toneladas en 1996 (Herrera, 1999).

v) Aguas negras y azolves. El rápido crecimiento de las ciudades ha propiciado la generación de aguas negras. En la cuenca de Pátzcuaro existen 23 descargas de aguas negras ubicadas en diferentes localidades, las que generan alrededor de 222 litros por segundo. Sin embargo, se calcula que entran al embalse 145 litros por segundo, debido a que dichas aguas se diluyen en el suelo antes de alcanzar la orilla, por la inexistencia de colectores, por el uso agrícola que se les da antes de desembocar, o bien, porque las cuatro plantas de tratamiento que existen en la zona presentan serias deficiencias. ${ }^{6}$

\section{IV}

\section{Atisbos de rescate de capital social y de sinergia con el gobierno estadual en la cuenca de Pátzcuaro}

\begin{abstract}
A pesar de la situación actual de la cuenca de Pátzcuaro, varios fenómenos recientes pueden ser atisbos de que está emergiendo nuevamente el capital social de los grupos subalternos, en sinergia con partes del estado de Michoacán, dando pie a una recuperación ambiental de la cuenca. Esos atisbos son los que se enumeran a continuación.
\end{abstract}

\section{Un gobierno estadual con amplia legitimidad}

Por primera vez el gobierno de Michoacán es ocupado por el izquierdista Partido de la Revolución Democrática (PRD). Su candidato Lázaro Cárdenas Batel llegó a la gobernación de Michoacán tras un amplio triunfo electoral y con una legitimidad que difícilmente hubiera obtenido otro partido.

Esta situación abrió a las comunidades campesinas, a la "sociedad civil organizada" y a muchos sectores sociales, la posibilidad de concretar proyectos incluyentes y en beneficio de los más desprotegidos. La política del actual gobierno así lo deja entrever, pues hasta ahora sus principales acciones han estado orientadas a atender asuntos fundamentales para el desarrollo local, como son

\footnotetext{
${ }^{6}$ Información proporcionada por el biólogo Armando Rivas en entrevista sobre el saneamiento del lago de Pátzcuaro.
} 
la salud, la educación, el medio ambiente y el desarrollo agropecuario.

El gobierno estadual parte de la tesis de que para garantizar el desarrollo social y económico es condición básica reconstruir primero el tejido social. "Reconstrucción y fortalecimiento de los tejidos sociales como fórmula primaria de acción frente a las carencias: tal es la vía que, bien diseñada y puesta en marcha, posibilitará que sean los propios sujetos sociales los que asuman en el mediano y largo plazos los controles plenos sobre su vida social, política, cultural y productiva. En la perspectiva referida, no hay lugar para el clientelismo o para el control corporativo, pues al potenciar las capacidades propias del ser comunitario se reducen o eliminan las posibilidades de convertirlo en simple "masa de manipulación'" (Plan estatal de desarrollo Michoacán, 2003-2008, s/f).

En esta política el gobierno estadual plantea la estrategia de sumar otra variable - como criterio de asignación de recursos - a la variable denominada "mayor pobreza": "la mayor proclividad o potencialidad de autoorganización y de desarrollo organizativo de la gente, pues de lo que se trata es de incidir en las posibilidades regenerativas del tejido social como fórmula o palanca de los subsecuentes procesos de desarrollo. Desde esta óptica, las zonas o regiones pobres que tengan una mayor densidad cultural, asociativa y organizativa (usamos aquí el concepto de organización en su sentido más amplio) deberán recibir montos críticos de inversión social, entendiendo por ello: a) que sean cuantitativamente significativos en relación al objetivo básico de la inversión; b) que tengan las características de ser inversiones sociales polivalentes y en cadena para que cumplan objetivos de integralidad; c) que tengan un componente importante para el fortalecimiento y desarrollo de las capacidades organizativas y de gestión de los sujetos sociales implicados" (Plan estatal de desarrollo Michoacán 2003-2008, s/f).

\section{La creación del Comité Intermunicipal}

El Comité Intermunicipal para la cuenca de Pátzcuaro fue establecido en el año 2000 por los presidentes municipales de Pátzcuaro, Erongarícuaro, Quiroga y Tzintzuntzan, con el propósito de fortalecer la coordinación de esfuerzos y así evitar la dispersión de recursos (Plan estatal de desarrollo Michoacán, 2003-2008, s/f).

Para apoyar las iniciativas comunitarias y con el objetivo de planificar todas las acciones gubernamentales, el actual gobierno estadual promovió la reactivación del Comité Intermunicipal. Para hacerlo, la Coordinación de Relaciones Interinstitucionales, así como la Secretaría de Planeación para el Desarrollo con el apoyo de los Servicios Alternativos para la Educación y el Desarrollo A.C. (SAED), examinaron la situación de los municipios y elaboraron un Programa de Desarrollo Regional para mejorar la conservación ambiental y el desarrollo de las comunidades en la región. $^{7}$

La metodología de trabajo utilizada por los SAED fue importante para conocer la situación de la zona y para propiciar la participación comunitaria. Hubo alrededor de 100 reuniones comunitarias y 92 asambleas, y se formaron comités de representantes en localidades que en muchos casos habían sido antes ignoradas en la planificación gubernamental. El trabajo que se realizó en todas estas instancias fue validado por ellas mismas y se tradujo en líneas de acción en el Programa Regional.

Como resultado de este trabajo, se formó un grupo de promotores comunitarios, pues se pensó que para lograr el entendimiento y desarrollo de los programas era preciso involucrar a las comunidades a través de personas de las mismas comunidades. Los promotores trabajaron para mantener viva la red de participantes de base. Se esperaba que el programa se financiara y se lanzara después de saber quiénes serían los nuevos alcaldes, en noviembre del 2004, para que las instituciones federales y estaduales dieran continuidad al plan.

\section{El apoyo financiero de la Fundación Gonzalo Río Arronte y la participación del Instituto Mexicano de Tecnología del Agua}

Una coyuntura que abre la posibilidad de revertir el deterioro de la cuenca e impulsar procesos de autogestión para su desarrollo ambiental es la presencia del IMTA y de la Fundación Gonzalo Río Arronte.

En febrero de 2003, el gobierno estadual de Michoacán, los gobiernos municipales integrantes del Comité Intermunicipal, la Fundación Gonzalo Río Arronte y el IMTA firmaron un convenio general de colaboración para llevar a cabo diversas acciones orientadas a la recuperación ambiental de la cuenca del lago de Pátzcuaro.

La novedad de ese convenio estriba en que pretende canalizar la acción institucional para mejorar el medio ambiente de la cuenca a partir de la solución de aspectos vinculados al agua. Por las condiciones actuales de la zona y particularmente por la mala calidad del agua del lago de Pátzcuaro, este recurso se ha vuelto un tema

\footnotetext{
${ }^{7}$ Véase http://www.laneta.apc.org/saed.htm.
} 
neurálgico que concentra la atención de los diferentes actores sociales.

En esta coyuntura, el trabajo previamente realizado por el gobierno estadual con el apoyo de los SAED, la reactivación del Comité Intermunicipal, el respaldo económico de la Fundación Gonzalo Río Arronte y la presencia del IMTA, generó un escenario propicio para potenciar el capital social en diferentes ámbitos y contribuir así a revertir las tendencias al deterioro en la cuenca.

\section{La participación de organizaciones no guberna- mentales}

Las organizaciones no gubernamentales (ONG) se han convertido en un actor importante. Por varios años han trabajado para rescatar los recursos naturales de la zona. Los lazos que han establecido entre ellas y hacia el interior de las comunidades de la cuenca les ha permitido generar una red de relaciones sociales en torno a las cuales se han construido alianzas de "puente y escalera", las que sin duda son una palanca para posibilitar la recuperación y fortalecimiento del capital social existente en la cuenca.

\section{a) Centro de Estudios Sociales y Ecológicos A. C. (CESE)}

Las raíces del CESE están ligadas al movimiento regional surgido en 1980-1981 para oponerse a la instalación de un centro de investigación sobre reactores nucleares en la comunidad indígena de Santa Fe de la Laguna. La misión institucional del CESE es contribuir a impulsar un proceso de desarrollo regional sustentable, a partir de: i) el fortalecimiento de sujetos sociales estratégicos; ii) el diseño y desarrollo de una estrategia para que la sociedad civil regional, fundamentalmente los sectores populares, tengan más injerencia en la formulación de políticas públicas; iii) la formulación de un programa de educación ambiental, y iv) la realización de estudios e investigaciones técnicas y sociales que contribuyan a dar respuestas específicas a problemas regionales. En los últimos años se ha puesto especial atención en medidas de educación ambiental, en el marco de una estrategia de educación ambiental para la cuenca de Pátzcuaro impulsada por el CESE (COEECO, 2002).

\section{b) Organización Ribereña contra la Contaminación del Lago de Pátzcuaro (ORCA) \\ Esta organización surgió del movimiento iniciado por el CESE durante 1980-1981 en Santa Fe de la Laguna; en un primer momento tuvo como objetivo asesorar y}

capacitar a los campesinos de la zona respecto a las desventajas de instalar reactores nucleares. Actualmente, la ORCA se ha dedicado a apoyar proyectos de autogestión campesina, capacitación, talleres participativos, construcción de estufas Lorena y asesoría a los pescadores. Sus integrantes son ampliamente conocidos en la cuenca y tienen gran capacidad de convocatoria.

\section{c) Grupo Interdisciplinario de Tecnología Rural Apropiada (GIRA)}

Se creó en 1985. Sus principales objetivos son investigar, desarrollar y difundir tecnología apropiada para que los recursos naturales del sector rural se usen con eficiencia y de manera social y ecológicamente armónica; desarrollar esquemas de ecoproducción basados en el uso económico y ambientalmente sustentable de los recursos naturales locales; servir como centro de información, demostración, capacitación y asesoría, e intercambiar, coordinar y difundir experiencias con instituciones, grupos y personas afines. Su principal ámbito de acción es la región purhépecha, aunque también existe interés de colaboración nacional e internacional. Cuenta con investigadores y profesionales de las áreas de agronomía, biología, ecología, física y diseño gráfico.

\section{d) Servicios Alternativos para la Educación y el Desarrollo (SAED)}

SAED es una asociación civil que tiene como una de sus funciones principales la realización de investigaciones técnicas y sociales vinculadas con el medio ambiente. Realiza trabajos en materia de promoción social y educación en la Meseta Purhépecha. Esta asociación, más que actuar como una ONG, ha prestado servicios de consultoría a diversas instancias gubernamentales, en Michoacán y en otros estados, como Guanajuato.

\section{Pequeñas agrupaciones campesinas lideradas por mujeres}

El surgimiento y la potenciación del capital social femenino en las comunidades rurales es un fenómeno interesante. Últimamente en la zona han aparecido organizaciones locales lideradas por mujeres que han iniciado procesos para potenciar las capacidades de sus integrantes y que, basándose en la red de relaciones locales, están adquiriendo una presencia significativa.

\section{a) Centro de Apoyo al Desarrollo de la Mujer Purhépecha (UARHI)}

Esta organización no gubernamental impulsa el desarrollo integral de la mujer purhépecha. Tiene su sede 
en la comunidad de Santa Fe de la Laguna y es dirigida por una mujer: Guadalupe Hernández Dimas. Busca colaborar con el desarrollo social y productivo de las mujeres purhépechas y crear conciencia en ellas sobre la recuperación y uso de espacios de autodesarrollo, promoviendo la participación comunitaria y la cooperación. ${ }^{8}$

El UARHI se aboca a la capacitación en las habilidades artesanales, para mejorar la calidad de los productos; a la formación y desarrollo de unidades productivas que permitan elevar la productividad y generar utilidades para las familias, y a la difusión de los derechos humanos, para que las mujeres purhépechas conozcan sus espacios de expresión y participación.

La líder ha sabido vincularse más allá del nivel comunitario, de tal suerte que actualmente ocupa un puesto en la Secretaría de Desarrollo Social del Estado. Su vínculo gubernamental y su experiencia le han permitido multiplicar las acciones de capacitación, de liderazgo entre las mujeres y de fortalecimiento institucional, así como los proyectos productivos.

\section{b) Juchari Uinapikua}

Otro ejemplo relevante es el grupo de once mujeres campesinas que integran la organización Juchari Uinapikua (Nuestra Fuerza). Esta organización surgió en septiembre del 2001 como resultado de diferentes talleres de reflexión sobre los problemas de la zona y el papel de las mujeres en el entorno de la cuenca. Sus integrantes realizan trabajo comunitario en cinco localidades de la cuenca: Cucuchuchu, Nocutzepo, Santa Ana Chapitiro, Tzentzenguaro y San Jerónimo Purenchechuaro.

El objetivo de esta organización es elevar la calidad de vida de las mujeres de la región. Para ello, ha trabajado en mejorar los procesos productivos artesanales del grupo y ha buscado un mejor mercado y mejores precios para sus productos, con miras a la obtención de ganancias más justas.

Un ejemplo ilustrativo es el de Herminia Domingo, de la comunidad de Cucuchuchu, que desde hace varios meses está organizando a mujeres de su comunidad para fabricar artesanías y lograr mejores precios en el mercado. También ha contribuido a que las mujeres tomen conciencia de sus derechos dentro de la familia y de sus posibilidades de desarrollo personal.
Otro caso relevante es el de Odilia Molina, una mujer de la comunidad de Nocutzepo que inició allí hace años — por iniciativa personal- una biblioteca pública. Su esfuerzo se ha visto recompensado con el apoyo de la comunidad y ha podido avanzar dentro y fuera de ella. El trabajo realizado a partir de esa labor la ha llevado a establecer nexos con jóvenes profesionales de su comunidad, entre los que se cuentan dos abogados que dan asesoría gratuita y dos maestros que promueven el intercambio cultural entre comunidades. Este grupo ha surgido de la misma comunidad $y$, por las necesidades existentes en ella, ha avanzado en la elaboración de una plataforma medioambiental que contempla agua potable, relleno sanitario y reforestación. No obstante, la implementación de dicha plataforma va muy lento, pues ha permanecido al margen de los partidos políticos y de las instituciones gubernamentales. 9

\section{c) El Centro de Atención y Desarrollo Cultural Colibrí}

Un ejemplo de las potencialidades del capital social lo ilustra Tania Calderón, una joven profesional originaria del municipio de Tzintzuntzan, quien comenzó hace varios años un trabajo voluntario de alfabetización de jóvenes que por diferentes razones no habían podido efectuar o terminar sus estudios en la zona.

Tania inició su labor como voluntaria dentro del municipio. Posteriormente, la gente se fue interesando y sumando a su propuesta educativa, la que se amplió a una escuela para padres, videos educativos, educación sexual e información vocacional, entre otros aspectos.

Los resultados de su trabajo llamaron la atención del presidente municipal de Tzintzuntzan, lo que llevó a establecer un acuerdo con el gobierno estadual. Actualmente existe en el poblado, además del programa de alfabetización para adultos, el Centro de Atención y Desarrollo Cultural Colibrí.

Cabe mencionar que la labor de Tania ha tenido un fuerte el impacto, debido en gran parte a su carisma y a su capacidad de vincularse con los jóvenes de su comunidad y también con los funcionarios estaduales.

\footnotetext{
${ }^{8}$ Información disponible en http://www.geocities.com/paginapurepecha2002/julio02.htm.
}

\footnotetext{
${ }^{9}$ Aunque Odilia Molina participó y ganó en las elecciones primarias del PRD para ser candidata a regidora en el municipio de Erongarícuaro, optó por retirarse debido a presiones partidistas.
} 


\section{Del atisbo a la concreción.}

\section{A manera de conclusiones}

Las comunidades de la cuenca de Pátzcuaro y los actores sociales presentes en ella tienen una significativa presencia. Han logrado establecer no solo una amplia red de relaciones, sino también diversas formas de capital social, algunas veces difíciles de ubicar por el complejo entramado social de la zona.

No sería errado afirmar que, en muchas ocasiones y ante la embestida gubernamental (tanto electorera como de programas), el capital social de la zona se encuentra aletargado, y solo se hace presente ante algún acontecimiento que pueda afectar (bien o mal) a las comunidades. De otra suerte solo funciona a nivel microsocial, es decir, ante las festividades, cosechas, desgracias y actos que impactan de manera inmediata a los actores sociales.

En este sentido, los atisbos de capital social señalados antes no son garantía de concreción. Para que lo fuesen, ante todo habría que modificar el entramado institucional para que a través de la red de actores sociales de la cuenca se pudiesen provocar cambios sustanciales en beneficio de la región. Las modificaciones tendrían que abarcar de inmediato por lo menos tres áreas:

i) El gobierno estadual: aun cuando una premisa básica de sus programas es la restauración del tejido social y la participación de la sociedad para alcanzar las metas de los programas, estos siguen llevándose a cabo bajo el viejo esquema paternalista; por lo tanto, están desvinculados de la política estadual y no logran articular las capacidades locales de las comunidades, de las organizaciones no gubernamentales o de otros sectores gubernamentales. Por lo tanto, el esfuerzo gubernamental por restaurar el tejido social — que podría ser el elemento reconstituyente del capital social - no avanza, lo que limita el impacto social de los programas. Para restablecer el tejido social es indispensable identificar e impulsar redes de capital social burocrático y reestructurar la forma de implementar los programas.

ii) El Instituto Mexicano de Tecnología del Agua: pese a su probada capacidad técnica, el IMTA no ha establecido una adecuada relación con los actores sociales de la zona, particularmente con las organizaciones no gubernamentales. En estas últimas encontró un abierto rechazo, por haber hecho caso omiso de los conocimientos acumulados por las comunidades en materia ambiental y de la reflexión sobre los éxitos y fracasos ocurridos en ellas durante los últimos veinte años. Se tiene incluso la percepción de que algunos de los trabajos que el IMTA propone ya se han experimentado en la zona. ${ }^{10}$ Además, se cuestiona la rigidez y verticalidad de los proyectos del Instituto, pues se considera que estos deberían tener flexibilidad suficiente para adaptarse a las necesidades reales de las comunidades (López Ramírez y Martínez Ruiz, 2003). Urge, pues, un cambio de estrategia para que el IMTA se articule realmente con los actores locales y recupere la experiencia de trabajo en la zona.

iii) Las organizaciones no gubernamentales: han tenido un papel importante en la cuenca, pero a la vez se han convertido en escuela de activistas provenientes de diferentes comunidades, lo que ha creado una fuerte dependencia de los líderes comunitarios respecto de las ONG. En nuestra opinión, esto inhibe las posibilidades de que emerjan iniciativas locales propias, pues las comunidades, como en otras ocasiones en que han sido clientela política de los partidos o las autoridades, también se han vuelto un importante medio para justificar a las ONG ante sus financistas: los mismos lazos de dependencia señalados hacen que, después de un tiempo, muchas de las ONG dejen de ser formadoras de capital humano y se conviertan en gestoras de las comunidades o sus proyectos. Así, “...el papel que adquieren los grupos o los líderes de las organizaciones al intermediar, no solo en esta relación, sino en todo el disputado terreno de las relaciones multiformes y complejas que se dan

\footnotetext{
${ }^{10}$ Un ejemplo muy ilustrativo de lo anterior es la propuesta del IMTA para la construcción de estufas Lorena, en circunstancias de que el Grupo Interdisciplinario de Tecnología Rural Apropiada (GIRA) lleva 15 años promoviendo, construyendo y evaluando las estufas elaboradas en la zona. Otro caso es la propuesta de cursos de cultura del agua en una cuenca en la que la gente está saturada de cursos, talleres y reuniones.
} 
entre el gobierno local y la ciudadanía, es el de 'gestores'. De esta forma, el gestor se constituye en un actor social y político central, intermedia las relaciones entre la ciudadanía y el gobierno local con el objetivo de hacer llegar las demandas de la primera al segundo y, al mismo tiempo, de controlar la respuesta a dichas demandas" (Treviño, 1999). Es necesario romper esta dependencia y potenciar las iniciativas locales a través de las redes organizativas para poder implementar proyectos de desarrollo.

En este contexto, los esfuerzos locales parecen estar teniendo cada vez más presencia como posibilidades de autogestión comunitaria. Esta situación, enmarcada en economías locales cada vez más deterioradas y vulnerables, así como en procesos migratorios en los que la mujer ha pasado a ocupar un papel significativo, antiguamente reservado para los varones, parece encontrar en los mecanismos de cooperación y solidaridad - grupal, barrial y comunitaria - un intersticio en el cual vislumbrar un futuro menos incierto para sus comunidades.

Es muy ilustrativo comprobar que en las comunidades consideradas existe una fuerte coincidencia entre quienes encabezan esos esfuerzos organizativos, tanto en su forma de relacionarse dentro de sus comunidades como en sus vínculos hacia el exterior, y en la articulación que sus organizaciones han logrado en el ámbito gubernamental y no gubernamental para el apoyo o financiamiento de sus proyectos. ${ }^{11}$

Cobra sentido entonces analizar detalladamente y entender mejor cómo se entrelazan los diferentes tipos de capital social existentes en las comunidades de la cuenca, conocer sus vínculos internos y externos, identificar sus redes sociales y potenciar su crecimiento, para que el capital social se pueda potenciar e incorporar a los procesos generales que ocurren en los ámbitos de gobierno y de participación social. Así se podrá fomentar la gestión integral de una cuenca a partir de la temprana formación de estructuras comunitarias y grupales, llevando a altos niveles de confianza que faciliten acciones comprometidas de todos los involucrados, bajo una rigurosa teoría de toma de decisiones colaborativa (Hooper, 2000).

Sin embargo, para hacerlo es indispensable una estrategia de trabajo que permita canalizar los esfuerzos en beneficio de la cuenca. Consideramos que, por diver-

\footnotetext{
11 De las dirigentes mencionadas, Guadalupe, Herminia y Tania tienen un trabajo en el gobierno que facilita mucho el entendimiento de procesos burocráticos y la tarea de vincular a sus comunidades con los programas de gobierno. Odilia, por su parte, trabaja en GIRA, una de las ONG con mayor reconocimiento en la zona y que, como señala Odilia, le ha permitido formarse para beneficio de su comunidad.
}

sas razones, el IMTA es una instancia capaz de activar el potencial del capital social:

- El IMTA disfruta de reconocimiento científico y técnico, y es ajeno a las dinámicas locales de clientelismo autoritario, compadrazgo político o complacencia gubernamental, lo que en cierta medida garantiza una actuación imparcial y objetiva ante los problemas ambientales que es preciso resolver.

- El apoyo financiero de la Fundación Gonzalo Río Arronte con que cuenta el IMTA da a este la posibilidad de marcar la pauta de los trabajos futuros $y$, en consecuencia, de establecer los mecanismos de participación. Sin embargo, insistimos en que es necesario reestructurar sus mecanismos de trabajo con respecto a las comunidades.

- En este sentido, es preciso establecer las reglas de un nuevo entramado institucional para que todas las acciones de gobierno sean transversales y los proyectos que se realicen tengan mayor impacto en la cuenca.

Por lo tanto, emprender un programa para la recuperación ambiental de la cuenca del lago de Pátzcuaro es algo que no puede estar desvinculado de los actores sociales que en ella habitan, particularmente las comunidades que se ubican en su territorio. En este sentido, garantizar que el programa ha de tener éxito y que se pueda incidir paulatinamente en la recuperación ambiental de la zona, equivale a considerar válidas las relaciones sociales existentes en las comunidades que se piense intervenir; pues aun cuando existan esfuerzos organizativos en ellas, si el programa no reconoce las dinámicas propias de las comunidades, los procesos de autogestión o si sólo se enfoca a atender o resolver ciertos temas, desconociendo las redes existentes y los liderazgos positivos de la región, no será posible lograr avances sustanciales en las acciones emprendidas.

"Lo anterior remite a la replicabilidad del capital social desde el nivel microsocial al macrosocial. Es decir, el capital social no es solamente un recurso que poseen los individuos en sus redes personales, sino también grupos y comunidades en una forma diferente: la de instituciones y sistemas complejos. El desarrollo económico se juega en la posibilidad de pasar de una vida comunitaria, basada en lazos de parentesco, a sociedades organizadas por instituciones formales. Esto significa que el capital social está presente en diversos grados y formas en las instituciones del Estado, el mercado y la sociedad civil" (Arriagada, Miranda y Pávez, 2004).

Un elemento clave para aquilatar el capital social previo de los grupos destinatarios es la capacidad de los 
programas de reconocer y respetar la trayectoria previa de las comunidades en sus iniciativas de desarrollo, y de sumarse a ella. Con tal fin, el análisis de las redes de capital social a nivel local debe tener la capacidad de discernir entre los grupos o individuos que, por diferentes razones, se ven forzados a cooperar, y aquellos que participan voluntariamente por iniciativa e interés propios.

En consecuencia, los programas destinados a la sociedad (sean de recuperación ambiental, desarrollo comunitario o superación de la pobreza) pueden crear, recuperar o potenciar capital social solo si se lo proponen explícitamente. Si los promotores del desarrollo (organizaciones no gubernamentales, gobiernos y agencias externas) realmente desean trabajar en beneficio de las comunidades y sus recursos, deberán volver su mirada a nuevos enfoques de trabajo con la sociedad. De lo contrario, seguiremos haciendo del desarrollo un mundo virtual.

\section{Bibliografía}

Adler-Lomnitz, L. (1998): Redes sociales, cultura y poder. Ensayos de antropología latinoamericana, México, D.F., Miguel Angel Porrúa/ Facultad Latinoamericana de Ciencias Sociales (FLACSO).

(2003): Cómo sobreviven los marginados, México, D.F., Siglo XXI editores.

Arriagada, I., F. Miranda y T. Pávez (2004): Lineamientos de acción para el diseño de programas de superación de la pobreza desde el enfoque del capital social. Guía conceptual y metodológica, serie Manuales, N ${ }^{\circ}$ 36, LC/L.2179-P, Santiago de Chile, Comisión Económica para América Latina y el Caribe (CEPAL). Publicación de las Naciones Unidas, $\mathrm{N}^{\circ}$ de venta: S.04.II.G.106.

Atria, R. (2003): Capital social: concepto, dimensiones y estrategias para su desarrollo, en R. Atria, M. Siles y otros (comps.), Capital social y reducción de la pobreza en América Latina y el Caribe: en busca de un nuevo paradigma, LC/G.2194-P, Santiago de Chile, Comisión Económica para América Latina y el Caribe (CEPAL). Publicación de las Naciones Unidas, $\mathrm{N}^{\circ}$ de venta: S.03.II.G.03.

Atria, R., M. Siles y otros (comps.) (2003): Capital social y reducción de la pobreza en América Latina y el Caribe: en busca de un nuevo paradigma, LC/G.2194-P, Santiago de Chile, Comisión Económica para América Latina y el Caribe (CEPAL). Publicación de las Naciones Unidas, $\mathrm{N}^{\circ}$ de venta: S.03.II.G.03.

Bebbington, A. (2005): Estrategias de vida y estrategias de intervención: el capital social y los programas de superación de la pobreza, en I. Arriagada (comp.), Aprender de la experiencia: el capital social en la superación de la pobreza, serie Libros de la CEPAL, № 86, LC/G.2275-P, Santiago de Chile, septiembre. Publicación de las Naciones Unidas, $\mathrm{N}^{\circ}$ de venta: S.05.II.G.93.

Berdegué, J.A. (2001): Cooperating to Compete: Associative Peasant Business Firms in Chile, Wageningen, Países Bajos, Universidad de Wageningen.

Bourdieu, P. (1980): Le capital social: notes provisoires, Actes de la recherche en sciences sociales, vol. 31, París, Centro de Sociología Europeo.

(2001): Las estructuras sociales de la economía, Buenos Aires, Ediciones Manantiales.

COEECO (Consejo Estatal de Ecología del Estado de Michoacán) (2002): Estrategias de educación, comunicación e información ambientales de Michoacán, Morelia, Michoacán.

Coleman, J. (1990): Foundations of Social Theory, Cambridge, Massachusetts, Belknap Press.

Durston, J. (2002): El capital social campesino en la gestión del desarrollo rural: díadas, equipos, puentes y escaleras, LC/G.2185-P, Santiago de Chile, Comisión Económica para América Latina y el Caribe (CEPAL). Publicación de las Naciones Unidas, $\mathrm{N}^{\mathrm{o}}$ de venta: S.02.II.G.74.

Fine, B. (2001): Social Capital vs. Social Theory: Political Economy and Social Science at the Turn of the Millenium, Routledge, Londres.
Foster, G. (1963): The dyadic contract in Tzintzuntzan II: patronclient relationship, American Anthropologist, New Series, vol. 65, $\mathrm{N}^{\circ}$ 6, Arlington, American Anthropological Association, diciembre.

Fox, J. (1996): How does civil society thicken? The political construction of social capital in rural Mexico, World Development, vol. $24, \mathrm{~N}^{\circ} 6$, Amsterdam, Elsevier, junio.

Fukuyama, F. (1999): La gran ruptura, Buenos Aires, Editorial Atlántida.

González de la Rocha, M. (2003): México: oportunidades y capital social, en I. Arriagada (comp.), Aprender de la experiencia. El capital social en la superación de la pobreza, serie Libros de la CEPAL, No 86, LC/G.2275-P, Santiago de Chile, Comisión Económica para América Latina y el Caribe (CEPAL). Publicación de las Naciones Unidas, $N^{\circ}$ de venta: S.05.II.G.93.

Granovetter, M. (1985): Economic action and social structure: the problem of embeddedness, American Journal of Sociology, vol. 91, No 3, Chicago, The University of Chicago Press, noviembre.

Herrera R., J.L. (1999): Propuesta para el desarrollo de un modelo de agricultura sustentable en la Cuenca del Lago de Pátzcuaro, Pátzcuaro, Michoacán, Centro de Estudios Sociales y Ecológicos.

Hooper, B. (2000): Integrated catchment watershed management. Disponible en www.catchment.com

(2001): Catchment management in Australia: a critique and a way forward. Disponible en www.catchment.com

INEGI (Instituto Nacional de Estadística, Geografía e Informática) (2000): Censo general de población y vivienda 2000, México, D.F.

López Ramírez, E. y J. Martínez Ruiz (2003): Alcances y limitaciones en la recuperación ambiental de la cuenca del lago de Pátzcuaro: la perspectiva de los actores. Informe Técnico, México, D.F., Instituto Mexicano de Tecnología del Agua, Coordinación de Tecnología de Comunicación, Participación e Información, Subcoordinación de Participación Social.

Mauss, M. (1979): Ensayo sobre los dones. Motivo y forma del cambio en las sociedades primitivas, Sociología y antropología, Madrid, Tecnos.

North, D. (1990): Institutions, Institutional Change and Economic Performance, Cambridge, Massachusetts, Cambridge University Press.

Peña, H. y M. Solanes (2003): Gobernabilidad efectiva del agua: acción a través de asociaciones en Sudamérica, Santiago de Chile, Comisión Económica para América Latina y el Caribe (CEPAL)/Asociación Mundial para el Agua.

Plan estatal de desarrollo Michoacán 2003-2008 (s/f). Disponible en http://www.michoacan.gob.mx/gobierno/pedm.pdf

Putnam, R. (1993): Making Democracy Work: Civic Traditions in Modern Italy, Princeton, Princeton University Press.

Robison, L., M. Siles y A. Schmid (2003): El capital social y la reducción de la pobreza: hacia un paradigma maduro, en 
R. Atria, M. Siles y otros (comps.), Capital social y reducción de la pobreza en América Latina y el Caribe: en busca de un nuevo paradigma, LC/G.2194-P, Santiago de Chile, Comisión Económica para América Latina y el Caribe (CEPAL). Publicación de las Naciones Unidas, $\mathrm{N}^{\mathbf{0}}$ de venta: S.03.II.G.03.

Salazar, G. (2001): Memoria histórica y capital social, en J. Durston (comp.), Capital social y políticas públicas en Chile: investigaciones recientes, serie Políticas sociales, $\mathrm{N}^{\circ}$ 55, LC/L.1606-P, Santiago de Chile, Comisión Económica para América Latina y el Caribe (CEPAL). Publicación de las Naciones Unidas, $\mathrm{N}^{\circ}$ de venta: S.01.II.G.147.

Treviño, A. (1999): Actores y organizaciones por el agua, Revista Ciudades, $\mathrm{N}^{\circ}$ 43, México, D.F., Red Nacional de Investigación Urbana, julio-septiembre.

Trigilia, C. (2003): Capital social y desarrollo local, El capital social. Instrucciones de uso, Buenos Aires, Fondo de Cultura Económica.

Woolcock, M. (1998): Social capital and economic development: toward a theoretical synthesis and policy framework, en Theory and Society, vol. 27, $\mathrm{N}^{\circ}$ 2, Nueva York, Springer. 